\title{
Polifarmácia: prevalência e fatores associados em usuários da atenção primária à saúde de um município do sul do Brasil
}

\author{
Polypharmacy: prevalence and associated factors in primary health care users in a \\ municipality of southern Brazil
}

\section{Polifarmacia: prevalencia y factores asociados en usuarios de atención primaria de salud en un municipio del sur de Brasil}

Amauri Braga Simonetti ${ }^{1 *}$, Lissandra Glusczak ${ }^{1}$, Everton Toigo Somensi ${ }^{1}$, Gustavo Olszanski Acrani ${ }^{1}$, Ivana Loraine Lindemann ${ }^{1}$.

\begin{abstract}
RESUMO
Objetivo: Verificar a prevalência e os fatores associados à polifarmácia entre usuários da atenção primária à saúde em um município do Rio Grande do Sul. Métodos: É um estudo transversal com coleta de dados a partir da aplicação de questionário. Foram avaliados aspectos sociodemográficos, de saúde e de comportamento. Foi realizada estatística descritiva, incluindo cálculo de prevalência com intervalo de confiança de 95\% (IC95) e de razões de prevalência (RP) brutas e ajustadas. Resultados: A prevalência de polifarmácia foi de 15,7\%, sendo 8\% em adultos até 59 anos e 33\% em idosos. Houve associação significativa de polfarmácia com idade, autopercepção negativa da saúde, depressão, diabetes mellitus, hipertensão, doença cardíaca, artrite/artrose e não consumo de bebida alcoólica. Conclusão: A polifarmácia é uma ocorrência frequente entre os usuários da atenção primária, principalmente idosos, e está associada a fatores comportamentais e de saúde. Estes resultados revelam a necessidade de implementação de estratégias que alertem a população sobre o uso apropriado de medicamentos para reduzir os riscos da polifarmácia.
\end{abstract}

Palavras-chave: Polifarmácia, Atenção Primária à Saúde, Envelhecimento, Epidemiologia.

\begin{abstract}
Objective: To verify the prevalence and factors associated with polypharmacy among users of primary health care in a municipality in Rio Grande do Sul. Methods: It is a cross-sectional study with data collection through the application of a questionnaire. Sociodemographic, health and behavioral aspects were evaluated. Descriptive statistics were performed, including prevalence calculation with a 95\% confidence interval $(\mathrm{Cl} 95 \%)$ and crude and adjusted prevalence ratios (PR). Results: The prevalence of polypharmacy was $15.7 \%, 8 \%$ in adults up to 59 years old and $33 \%$ in the elderly. There was a significant association of polypharmacy with age, negative self-perceived health, depression, diabetes mellitus, hypertension, heart disease, arthritis/arthrosis and no alcohol consumption. Conclusion: Polypharmacy is a frequent occurrence among primary care users, especially the elderly, and is associated with behavioral and health factors. These results reveal the need to implement strategies that alert the population to the appropriate use of medicines to reduce the risks of polypharmacy.
\end{abstract}

Keywords: Polypharmacy, Primary Health Care, Aging, Epidemiology.

\section{RESUMEN}

Objetivo: Verificar la prevalencia y los factores asociados a la polifarmacia entre los usuarios de la atención primaria de salud en um municipio de Rio Grande do Sul. Métodos: Es un estudio transversal con recolección de datos mediante la aplicación de un cuestionario. Se evaluaron aspectos sociodemográficos, de salud y conductuales. Se realizaron estadísticas descriptivas, incluido el cálculo de la prevalencia con un intervalo de confianza del 95\% (IC $95 \%$ ) y razones de prevalencia (RP) brutas y ajustadas. Resultados: La prevalencia de polifarmacia fue de 15,7\%, $8 \%$ en adultos hasta 59 años y $33 \%$ en ancianos. Hubo una asociación significativa de polifarmacia con edad, salud autopercibida negativa, depresión, diabetes mellitus, hipertensión, enfermedades cardíacas, artritis/artrosis y no consumo de bebidas alcohólicas. Conclusión: La polifarmacia es una ocurrencia frecuente entre los usuarios de atención primaria, especialmente los ancianos, y está asociada a factores conductuales y de salud. Estos resultados revelan la necesidad de implementar estrategias que alerten a la población sobre el uso adecuado de medicamentos para reducir los riesgos de polifarmacia.

Palabras clave: Polifarmacia, Atención Primaria en Salud, Envejecimiento, Epidemiología.

${ }^{1}$ Universidade Federal da Fronteira Sul (UFFS), Passo Fundo - RS. *E-mail: amauri.simonetti@uffs.edu.br 


\section{INTRODUÇÃO}

O Sistema Único de Saúde (SUS), criado pela Constituição Federal, tem como foco a Atenção Primária à Saúde (APS) que inclui um conjunto de ações, no contexto individual e coletivo, englobando promoção e proteção da saúde, prevenção, diagnóstico e tratamento de doenças, reabilitação e redução de danos (BRASIL, 2020). No município de Passo Fundo, localizado ao norte do Rio Grande do Sul (RS), com uma população estimada de 204.722 habitantes (IBGE, 2020), a APS é constituída por Unidades Básicas de Saúde (UBS), Estratégias de Saúde da Família (ESF), Centros de Atendimento Integral à Saúde (CAIS) e Centros de Atenção Psicossocial (CAPS) (PASSO FUNDO, 2020).

Nos últimos anos, o consumo de medicamentos pela população tem aumentado substancialmente, principalmente devido às doenças crônicas e às manifestações clínicas decorrentes do envelhecimento (STEFANO ICA, et al., 2017). A influência exercida pela indústria farmacêutica, a facilidade de obtenção, a tendência de automedicação na sociedade, a falta de conscientização para a utilização de medidas não farmacológicas e, até mesmo, o uso contraindicado de fármacos por profissionais da saúde, podem contribuir para essa realidade (SILVEIRA EA, et al., 2014; LUTZ BH, et al., 2017; COSTA GM, et al., 2017).

A polifarmácia é um fenômeno cada vez mais frequente entre os idosos e pode causar sérias complicações, como interações medicamentosas e reações adversas aos medicamentos, além de prejuízos econômicos ao SUS devido aos gastos com internações (SECOLI SR, et al., 2012; WHO, 2019).

A polifarmácia é descrita como o uso concomitante de vários medicamentos e, embora haja controvérsia sobre o número, a definição mais empregada se refere à utilização de 5 ou mais medicamentos de uso contínuo (MASNOON N, et al., 2017). Esse evento tem sido relatado em diversos estudos, principalmente em idosos, mas somente alguns mais recentes analisaram a sua prevalência no contexto da APS e em sistemas públicos de saúde (FLORES LM e MENGUE SS, 2005; MARIN MJS, et al., 2008; SILVEIRA EA, et al., 2014; GUTHRIE B, et al., 2015; COSTA GM, et al., 2017; OLIVEIRA PC, et al., 2019).

Em um inquérito recente, integrante da Pesquisa Nacional sobre o Acesso, Utilização e Promoção do Uso Racional de Medicamentos no Brasil (PNAUM), foram entrevistados 8.803 usuários de serviços de APS em 272 municípios brasileiros, sendo encontrada uma prevalência de polifarmácia em 9,4\% da população geral e de $18,1 \%$ em idosos, associada com variáveis sociodemográficas e indicadores de saúde (NASCIMENTO RCRM, et al., 2017).

No Rio Grande do Sul (RS) são escassos os estudos sobre polifarmácia (FLORES LM e MENGUE SS, 2005; LUTZ BH, et al., 2017). De conhecimento dos autores, até o presente momento foram relatados somente duas pesquisas na região noroeste e norte do estado, sendo uma no município de Santa Rosa e o outro em Coxilha, porém, ambos através de entrevista domiciliar. Este é o primeiro estudo no âmbito da APS na região de Passo Fundo, que é o maior polo médico do interior do estado (FLORES VB e MENGUE SS, 2008; CAVALCANTI G, et al., 2017).

Neste contexto, o objetivo desta pesquisa foi verificar a prevalência e os fatores associados à polifarmácia em usuários atendidos na rede de APS em um município da região norte do RS.

\section{MÉTODOS}

Este estudo faz parte de uma pesquisa transversal mais ampla realizada com adultos e idosos atendidos na rede urbana de APS de Passo Fundo, norte do estado do Rio Grande do Sul. O cálculo amostral para a referida pesquisa foi feito de duas formas, com nível de confiança de $95 \%$ e poder de $80 \%$ para ambas. No primeiro, para identificar prevalência mínima de $10 \%$ de qualquer desfecho de interesse, admitida margem de erro de cinco pontos percentuais, obteve-se tamanho amostral de 138. No segundo, para verificar a associação entre os diferentes desfechos e variáveis preditoras, considerando 9 não expostos para cada exposto, prevalência total do desfecho de $10 \%$, frequência esperada em não expostos de $9,1 \%$ e, RP de 2 , constatou-se a necessidade de 1.220 participantes. Acrescentando-se a esse número $15 \%$ para fatores de confusão, definiu-se a amostra em 1.403.

As 34 unidades de saúde foram abrangidas e o quantitativo em cada local foi proporcional ao número de procedimentos no mês anterior, sendo que todos os usuários que aguardavam consulta foram incluídos até que se atingisse o número definido. Consideraram-se elegíveis indivíduos com idade igual ou superior a 18 
anos, de ambos os sexos, residentes na cidade e excluíram-se acamados, portadores de alguma deficiência que os impedisse de responder ao questionário e, para este estudo, também as gestantes. Os dados foram coletados entre maio e agosto de 2019, a partir da aplicação de questionário padronizado nas próprias unidades.

O desfecho, polifarmácia, foi aferido por meio da pergunta: Tem algum remédio que você toma todos os dias? Em caso de resposta afirmativa foi perguntado: Quantos remédios você toma todos os dias? Aqueles que referiram uso de 5 ou mais fármacos diariamente foram classificados como polimedicados (MASNOON N, et al., 2017).

As variáveis de exposição abrangeram sexo, idade, cor da pele referida, escolaridade, situação conjugal, condições de moradia, exercício de atividade remunerada e renda familiar mensal per capita em salários mínimos (R\$998,00). Avaliou-se ainda costume de pesquisar sobre saúde na internet, tabagismo, consumo de bebida alcoólica, prática de atividade física e automedicação (consumo de medicamento por conta própria, sem receita, nos 30 dias anteriores à coleta de dados).

Também foi avaliado autopercepção da saúde, multimorbidade (considerando duas ou mais doenças crônicas por diagnóstico médico autorreferido de obesidade, diabetes mellitus, hipertensão arterial sistêmica, hipercolesterolemia, hipertrigliceridemia e doença cardíaca), diagnóstico médico referido de diabetes mellitus, hipertensão arterial sistêmica, hipercolesterolemia, hipertrigliceridemia, doença cardíaca e da tireoide, depressão, câncer, alergia e artrite ou artrose, dor crônica (duração de 6 meses ou mais) e vigência de tratamento psicológico. Além disso, o excesso de peso foi determinado a partir de peso e altura referidos e classificação pelo Índice de Massa Corporal (LIPSCHITZ DA, 1994; WHO, 1995; WHO, 2009).

Os dados foram duplamente digitados e validados e a análise estatística incluiu as frequências absolutas e relativas para caracterização amostral, além do cálculo da prevalência do desfecho com intervalo de confiança de 95\% (IC95). As associações entre a polifarmácia e os fatores de exposição foram verificadas na análise bivariada, a partir das razões de prevalência (RP) brutas e seus IC95. Na análise multivariada foi realizado controle para fatores de confusão sendo que as RP ajustadas e seus IC95 foram gerados por Regressão de Poisson com variância robusta para conglomerados. A análise foi do tipo backward stepwise seguindo um modelo hierárquico pré-definido com três níveis de determinação (VICTORA CG, et al., 1997).

No $1^{\circ}$ nível foram incluídas as variáveis sociodemográficas, no $2^{\circ}$ as de saúde e, no $3^{\circ}$ nível, as variáveis comportamentais. Em cada nível as variáveis foram ajustadas entre si e aquelas que apresentaram $p \leq 0,20$ foram mantidas para ajuste com o nível seguinte. No caso das categóricas politômicas, quando houve ordenamento entre as categorias foi realizado o teste de Wald para tendência linear e, quando não houve ou não foi significativo, foi testada a heterogeneidade. Em todos os testes, foi admitido erro a de $5 \%$, sendo considerados significativos valores de $p<0,05$, para testes bicaudais.

O protocolo do estudo foi aprovado pelo Comitê de Ética em Pesquisa com Seres Humanos da Universidade Federal da Fronteira Sul, obedecendo à Resolução no 466/2012 do Conselho Nacional de Saúde (Parecer de no 3.219.633).

\section{RESULTADOS}

Foram amostrados 1.365 participantes, sendo observado predomínio de sexo feminino (69,4\%), idade entre 18 e 59 anos (70,4\%), cor da pele branca (64,8\%), até 8 anos de estudo (47,1\%), indivíduos com cônjuge $(71,3 \%)$, que não moram sozinhos $(90 \%)$, não exercem atividade remunerada $(57,7 \%)$ e com renda de até um salário mínimo (70,7\%). Do total, 52,1\% apresentaram autopercepção positiva da saúde e 65\%, excesso de peso.

Quanto ao diagnóstico médico de doenças crônicas não transmissíveis, 42,3\% mencionaram duas ou mais, sendo 19,9\% diabetes mellitus, 40,9\% de hipertensão arterial sistêmica, 26,1\% de hipercolesterolemia, $19,8 \%$ de hipertrigliceridemia, 15,2\% de doença cardíaca e 14,9\% da tireoide, 27,6\% de depressão, 5,4\% de câncer, $31 \%$ de alergia e $17,4 \%$ de artrite/artrose. Dor crônica foi referida por $56,1 \%$ dos participantes e tratamento psicológico por 8,7\%. Ainda, 63,8\% referiram costume de pesquisar sobre saúde na internet, $18,9 \%$ relataram tabagismo, 30,3\% consumo de bebida alcoólica, 50,9\% automedicação e, 56,9\%, que não praticavam atividade física (Tabela 1). 
Tabela 1 - Caracterização de uma amostra de adultos e idosos atendidos na Atenção Primária à Saúde.

\begin{tabular}{|c|c|c|}
\hline Variáveis & $\mathbf{n}$ & $\%$ \\
\hline \multicolumn{3}{|l|}{ Sexo } \\
\hline Feminino & 947 & 69,4 \\
\hline Masculino & 418 & 30,6 \\
\hline \multicolumn{3}{|c|}{ Idade em anos completos $(n=1.361)$} \\
\hline $18-59$ & 958 & 70,4 \\
\hline$\geq 60$ & 403 & 29,6 \\
\hline \multicolumn{3}{|l|}{ Cor da pele referida $(n=1.359)$} \\
\hline Outras & 478 & 35,2 \\
\hline Branca & 881 & 64.8 \\
\hline \multicolumn{3}{|l|}{ Escolaridade em anos $(n=1.261)$} \\
\hline$\leq 8$ & 594 & 47,1 \\
\hline $9-11$ & 415 & 32.9 \\
\hline$\geq 12$ & 252 & 20.0 \\
\hline \multicolumn{3}{|l|}{ Situação conjugal $(n=1.358)$} \\
\hline Com cônjuge & 968 & 71,3 \\
\hline Sem cônjuge & 390 & 28,7 \\
\hline \multicolumn{3}{|l|}{ Mora sozinho } \\
\hline Não & 1.228 & 90,0 \\
\hline Sim & 137 & 10.0 \\
\hline \multicolumn{3}{|l|}{ Exercício de atividade remunerada } \\
\hline Sim & 577 & 42,3 \\
\hline Não & 788 & 57,7 \\
\hline \multicolumn{3}{|c|}{ Renda familiar mensal per capita* $(n=1.278)$} \\
\hline$\leq 1$ & 904 & 70,7 \\
\hline$>1$ & 374 & 29,3 \\
\hline \multicolumn{3}{|l|}{ Autopercepção da saúde ( $\mathrm{n}=1.354)$} \\
\hline Positiva & 706 & 52,1 \\
\hline Negativa & 648 & 47.9 \\
\hline \multicolumn{3}{|l|}{ Agravos à saúde } \\
\hline Multimorbidade & 577 & 42,3 \\
\hline Excesso de peso $(n=1.196)$ & 777 & 65,0 \\
\hline Diabetesmellitus ${ }^{\star \star}$ & 271 & 19,9 \\
\hline Hipertensão arterial sistêmica** & 558 & 40,9 \\
\hline Hipercolesterolemia** & 357 & 26,1 \\
\hline Hipertrigliceridemia** & 271 & 19,8 \\
\hline 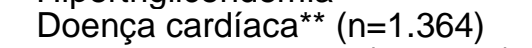 & 207 & 15,2 \\
\hline Doença da tireoide ${ }^{\star \star}(n=1.364)$ & 203 & 14,9 \\
\hline Depressão** & 376 & 27,6 \\
\hline Câncer** $(n=1.364)$ & 73 & 5,4 \\
\hline 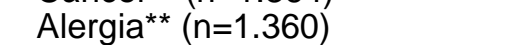 & 422 & 31,0 \\
\hline Artrite/artrose ${ }^{* *}(n=1.362)$ & 237 & 17,4 \\
\hline Dor crônica $(n=777)$ & 436 & 56,1 \\
\hline \multicolumn{3}{|l|}{ Tratamento psicolóqico $(\mathrm{n}=1.363)$} \\
\hline Não & 1.244 & 91,3 \\
\hline Sim & 119 & 8,7 \\
\hline \multicolumn{3}{|c|}{ Costuma pesquisar sobre saúde na internet ( $n=923)$} \\
\hline Sim & 589 & 63,8 \\
\hline Não & 334 & 36,2 \\
\hline \multicolumn{3}{|l|}{ Tabagismo $(n=1.363)$} \\
\hline Sim & 257 & 18,9 \\
\hline Não & 1.106 & 81,1 \\
\hline \multicolumn{3}{|c|}{ Consumo de bebida alcoólica ( $n=1.364)$} \\
\hline Sim & 414 & 30.3 \\
\hline Não & 950 & 69,7 \\
\hline \multicolumn{3}{|l|}{ Prática de atividade física $(n=1.364)$} \\
\hline Não & 776 & 56,9 \\
\hline $\operatorname{Sim}$ & 588 & 43,1 \\
\hline \multicolumn{3}{|l|}{ Automedicação } \\
\hline Não & 670 & 49,1 \\
\hline Sim & 695 & 50,9 \\
\hline
\end{tabular}

Legenda: * Em salários mínimos: $\mathrm{R} \$ 998,00 ;{ }^{* \star}$ Diagnóstico médico referido.

Fonte: Simonetti ABS, et al., 2021. 
Observou-se prevalência de polifarmácia de 15,7\% (IC95 14-18) na amostra, sendo 8\% (IC95 7-10) em adultos até 59 anos e 33\% (IC95 28-37) em idosos acima de 60 anos. Mesmo após ajuste, maior probabilidade do desfecho foi observada em idosos ( $R P=2,75$; IC95 2,01-3,74), participantes que não estavam trabalhando $(\mathrm{RP}=1,86$; IC95 1,22-2,84), com autopercepção negativa da saúde ( $\mathrm{RP}=2,00 ; \mathrm{IC95}$ 1,39-2,88), diabéticos ( $\mathrm{RP}=2,62$; IC95 2,00-3,43), hipertensos ( $\mathrm{RP}=3,77$; IC95 2,64-5,38), com doença cardíaca (RP=1,60; IC95 $1,30-1,98)$, depressão ( $R P=1,47$; IC95 1,19-1,82), artrite/artrose $(R P=1,50 ; \operatorname{IC95} 1,25-180)$ e que não consumiam bebida alcoólica $(\mathrm{RP}=1,41$; IC95 1,08-1,82). Por outro lado, foi observada uma menor probabilidade do desfecho entre aqueles que se automedicaram nos últimos 30 dias ( $R P=0,76$; IC95 0,590,97) (Tabelas 2, 3 e 4).

Tabela 2 - Análise bruta e ajustada de fatores associados à polifarmácia em adultos e idosos atendidos na Atenção Primária à Saúde, $1^{\circ}$ nível: variáveis sociodemográficas. Passo Fundo, RS, 2019. ( $\left.n=1.174\right)$.

\begin{tabular}{|c|c|c|c|c|}
\hline Variáveis & RP Bruta (IC95) & $\mathbf{p}$ & RP Ajustada (IC95) & $\mathbf{p}$ \\
\hline Sexo & & $0,162^{a}$ & & $0,843^{a}$ \\
\hline Masculino & 1,00 & & 1,00 & \\
\hline Feminino & $1,15(0,95-1,39)$ & & $1,03(0,80-1,32)$ & \\
\hline Idade em anos completos & & $<0,001^{a}$ & & $<0,001^{a}$ \\
\hline $18-59$ & 1,00 & & 1,00 & \\
\hline$\geq 60$ & $3,87(2,96-5,07)$ & & $2,75(2,01-3,74)$ & \\
\hline Cor da pele autorreferida & & $0,172^{\mathrm{a}}$ & & $0,158^{a}$ \\
\hline Outras & 1,00 & & 1,00 & \\
\hline Branca & $1,15(0,94-1,40)$ & & $1,18(0,94-1,49)$ & \\
\hline Escolaridade em anos & & $<0,001^{b}$ & & $0,185^{b}$ \\
\hline$\leq 8$ & 1,00 & & 1,00 & \\
\hline $9-11$ & $0,56(0,42-0,73)$ & & $0,79(0,60-1,04)$ & \\
\hline$\geq 12$ & $0,57(0,38-0,84)$ & & $0,84(0,54-1,29)$ & \\
\hline Situação conjugal & & $0,047^{a}$ & & $0,423^{a}$ \\
\hline Com cônjuge & 1,00 & & 1,00 & \\
\hline Sem cônjuge & $1,23(1,00-1,51)$ & & $1,09(0,89-1,34)$ & \\
\hline Morar sozinho & & 0,001 & & 0,895 \\
\hline Não & 1,00 & & 1,00 & \\
\hline Sim & $1,63(1,24-2,15)$ & & $1,03(0,66-1,62)$ & \\
\hline $\begin{array}{l}\text { Exercício de atividade } \\
\text { remunerada }\end{array}$ & & $<0,001^{a}$ & & $0,004^{a}$ \\
\hline Sim & 1,00 & & 1,00 & \\
\hline Não & $3,29(2,26-4,78)$ & & $1,86(1,22-2,84)$ & \\
\hline $\begin{array}{l}\text { Renda familiar mensal per capita } \\
\text { em salários mínimos }{ }^{\star *}\end{array}$ & & $0,553^{a}$ & & $0,797^{a}$ \\
\hline$\leq 1$ & 1,00 & & 1,00 & \\
\hline$>1$ & $1,09(0,83-1,43)$ & & $1,04(0,75-1,46)$ & \\
\hline
\end{tabular}

Legenda: *Diagnóstico médico referido; ${ }^{* *} \mathrm{R} \$ 998,00$ aQui-quadrado; ${ }^{\mathrm{b}}$ Heterogeneidade.

Fonte: Simonetti ABS, et al., 2021. 
Tabela 3 - Análise bruta e ajustada de fatores associados à polifarmácia em adultos e idosos atendidos na Atenção Primária à Saúde, $2^{\circ}$ nível: variáveis de saúde. Passo Fundo, RS, 2019. (n=622).

\begin{tabular}{|c|c|c|c|c|}
\hline Variáveis & RP Bruta (IC95) & $\mathbf{p}$ & RP Ajustada (IC95) & $\mathbf{p}$ \\
\hline Autopercepção da saúde & & $<0,001^{a}$ & & $<0,001^{\mathrm{a}}$ \\
\hline Positiva & 1,00 & & 1,00 & \\
\hline Negativa & $4,57(3,33-6,27)$ & & $2,00(1,39-2,88)$ & \\
\hline Multimorbidade & & $<0,001^{a}$ & & $0,608^{a}$ \\
\hline Não & 1,00 & & 1,00 & \\
\hline Sim & $9,07(6,60-12,47)$ & & $1,27(0,51-3,21)$ & \\
\hline Excesso de peso & & $0,006^{a}$ & & $0,293^{a}$ \\
\hline Não & 1,00 & & 1,00 & \\
\hline Sim & $1,46(1,12-1,90)$ & & $1,17(0,87-1,57)$ & \\
\hline Diabetes mellitus ${ }^{\star}$ & & $<0,001^{\mathrm{a}}$ & & $<0,001^{a}$ \\
\hline Não & 1,00 & & 1,00 & \\
\hline Sim & $5,56(4,30-7,20)$ & & $2,62(2,00-3,43)$ & \\
\hline $\begin{array}{l}\text { Hipertensão arterial } \\
\text { sistêmica* }\end{array}$ & & $<0,001^{\mathrm{a}}$ & & $<0,001^{a}$ \\
\hline Não & 1,00 & & 1,00 & \\
\hline Sim & $9,23(6,86-12,41)$ & & $3,77(2,64-5,38)$ & \\
\hline Hipercolesterolemia* & & $<0,001^{a}$ & & $0,328^{a}$ \\
\hline Não & 1,00 & & 1,00 & \\
\hline Sim & $3,47(2,60-4,63)$ & & $1,14(0,87-1,49)$ & \\
\hline Hipertrigliceridemia* & & $<0,001^{a}$ & & $0,999^{a}$ \\
\hline Não & 1,00 & & 1,00 & \\
\hline Sim & $3,41(2,73-4,26)$ & & $1,00(0,75-1,34)$ & \\
\hline Doença cardíaca* $^{*}$ & & $<0,001^{a}$ & & $<0,001^{a}$ \\
\hline Não & 1,00 & & 1,00 & \\
\hline Sim & $4,38(3,50-5,47)$ & & $1,60(1,30-1,98)$ & \\
\hline Doença da tireoide $^{\star}$ & & $<0,001^{a}$ & & $0,493^{a}$ \\
\hline Não & 1,00 & & 1,00 & \\
\hline Sim & $2,03(1,66-2,48)$ & & $1,08(0,87-1,32)$ & \\
\hline Depressão* & & $<0,001^{a}$ & & $<0,001^{a}$ \\
\hline Não & 1,00 & & 1,00 & \\
\hline Sim & $2,49(1,94-3,18)$ & & $1,47(1,19-1,82)$ & \\
\hline Câncer* & & $0,007^{a}$ & & $0,414^{\mathrm{a}}$ \\
\hline Não & 1,00 & & 1,00 & \\
\hline Sim & $1,93(1,20-3,12)$ & & $0,84(0,56-1,27)$ & \\
\hline Alergia* & & $0,921^{\mathrm{a}}$ & & $0,726^{a}$ \\
\hline Não & 1,00 & & 1,00 & \\
\hline Sim & $1,01(0,78-1,31)$ & & $1,04(0,84-1,28)$ & \\
\hline Artrite/artrose & & $<0,001^{a}$ & & $<0,001^{a}$ \\
\hline Não & 1,00 & & 1,00 & \\
\hline Sim & $3,32(2,54-4,33)$ & & $1,50(1,25-1,80)$ & \\
\hline Dor crônica & & $<0,001^{a}$ & & $0,491^{a}$ \\
\hline Não & 1,00 & & 1,00 & \\
\hline Sim & $2,06(1,58-2,69)$ & & $1,09(0,85-1,41)$ & \\
\hline Tratamento psicológico & & $<0,001^{a}$ & & $0,506^{a}$ \\
\hline Não & 1,00 & & 1,00 & \\
\hline Sim & $1,84(1,35-2,51)$ & & $1,12(0,80-1,57)$ & \\
\hline
\end{tabular}

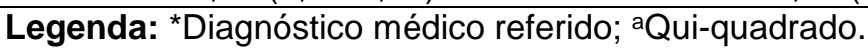

Fonte: Simonetti ABS, et al., 2021. 
Tabela 4 - Análise bruta e ajustada de fatores associados à polifarmácia em adultos e idosos atendidos na Atenção Primária à Saúde, 3onível: variáveis comportamentais. Passo Fundo, RS, 2019. $(n=873)$.

\begin{tabular}{|c|c|c|c|c|}
\hline Variáveis & RP Bruta (IC95) & $\mathbf{p}$ & RP Ajustada (IC95) & $\mathbf{p}$ \\
\hline $\begin{array}{l}\text { Costume de pesquisar sobre } \\
\text { saúde na internet }\end{array}$ & & $0,115^{\mathrm{a}}$ & & $0,976^{a}$ \\
\hline Sim & 1,00 & & 1,00 & \\
\hline Não & $1,42(0,92-2,19)$ & & $0,99(0,65-1,51)$ & \\
\hline Tabagismo & & $0,176^{a}$ & & $0,546^{a}$ \\
\hline Sim & 1,00 & & 1,00 & \\
\hline Não & $1,32(0,88-1,98)$ & & $1,13(0,76-1,67)$ & \\
\hline Consumo de bebida alcoólica & & $<0,001^{a}$ & & $0,010^{a}$ \\
\hline Sim & 1,00 & & 1,00 & \\
\hline Não & $1,96(1,44-2,65)$ & & $1,41(1,08-1,82)$ & \\
\hline Prática de atividade física & & $0,412^{a}$ & & $0,577^{a}$ \\
\hline Não & 1,00 & & 1,00 & \\
\hline $\operatorname{Sim}$ & $1,11(0,86-1,45)$ & & $0,94(0,75-1,17)$ & \\
\hline $\begin{array}{l}\text { Automedicação nos últimos } \\
30 \text { dias }\end{array}$ & & $0,020^{a}$ & & $0,029^{a}$ \\
\hline Não & 1,00 & & 1,00 & \\
\hline Sim & $0,73(0,56-0,95)$ & & $0,76(0,59-0,97)$ & \\
\hline
\end{tabular}

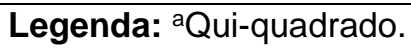

Fonte: Simonetti ABS, et al., 2021.

\section{DISCUSSÃO}

Poucos estudos foram encontrados na literatura nacional e internacional no contexto da APS para a análise da prevalência de polifarmácia e seus fatores associados, porém, um fato comum é a utilização de múltiplos medicamentos entre idosos (FLORES LM e MENGUE SS, 2005; MARIN MJS, et al., 2008; GRIMMSMANN T, et al., 2009; SILVEIRA EA, et al., 2014; GUTHRIE B, et al., 2015; COSTA GM, et al., 2017; NASCIMENTO RCRM, et al., 2017; OLIVEIRA PC, et al., 2019).

A prevalência de polifarmácia observada foi de $15,7 \%$, sendo este valor superior ao verificado de $9,4 \%$ em um grande estudo brasileiro com usuários de serviços de saúde nas cinco regiões do Brasil (NASCIMENTO RCRM, et al., 2017) e de 10\% em outro realizado na Alemanha (GRIMMSMANN T, et al., 2009). Por outro lado, o valor é inferior ao observado (27\%) em Porto Alegre, RS (FLORES LM e MENGUE SS, 2005) eo relatado (20,8\%) na Escócia (GUTHRIE B, et al., 2015).

$\mathrm{Na}$ população em geral, a literatura aborda a ocorrência de polifarmacoterapia em idosos devido à tendência de maior utilização de fármacos, principalmente para as doenças crônicas. Considerando que, idoso é o indivíduo com 60 anos ou mais de idade, neste trabalho a prevalência de polifarmácia foi de 33\%, valor semelhante aos $32,7 \%$ verificados na cidade do Rio de Janeiro e aos $32 \%$ em Florianópolis superior às frequências verificadas em algumas regiões do país, com variação de 29,4\% a 10,3\% (ROZENFELD S, et al., 2008; PEREIRA KG, et al., 2017; LOYOLA FILHO I, et al., 2006; SILVEIRA EA, et al., 2014; RAMOS LR, et al., 2016; COSTA GM, et al., 2017; NASCIMENTO RCRM, et al., 2017; ALMEIDA NA, et al., 2017; MARQUES $\mathrm{PP}$, et al., 2019) e inferior às relatadas por outros autores, com valores de $35,3 \%$ a $57,7 \%$ (CAVALCANTI G, et al., 2017; OLIVEIRA PC, et al., 2019).

A variabilidade na prevalência observada entre os estudos pode ser devida ao tamanho da amostra, tipo de coleta de dados (entrevista domiciliar ou em serviços de saúde, coleta em prontuário médico ou por autorreferimento), populações com diferentes etnias e hábitos de vida, condições socioeconômicas, utilização de serviços e modelo de atenção à saúde, além de, características demográficas regionais e culturais ligadas ao consumo de medicamentos (LOYOLA FILHO I, et al., 2006; NASCIMENTO RCRM, et al., 2017). 
No Brasil a automedicação é um fator importante a considerar, provavelmente pela facilidade de acesso a medicamentos e pela falta de conscientização para a adesão a medidas não farmacológicas. Embora 50,9\% dos participantes desta pesquisa tenham referido a prática, menor probabilidade de polifarmácia ocorreu entre aqueles que se automedicaram nos últimos 30 dias (redução de 24\%).Na literatura há uma heterogeneidade de resultados com valores de $16,1 \%$ a $35 \%$ de automedicação, conforme o período recordatório analisado dos últimos sete ou quinze dias, em diferentes regiões do Brasil e características sociodemográficas diversas (DOMINGUES PHF, et al., 2015; ARRAIS PSD, et al., 2016).

No estudo PNAUM foram entrevistados pacientes em 1.305 serviços de APS nas cinco regiões do Brasil. Destes, $76,2 \%$ relataram ter utilizado algum medicamento nos 30 dias anteriores à entrevista, com número médio de medicamentos de 2,3 (NASCIMENTO RCRM, et al., 2017). Pela facilidade de acesso a medicamentos, medicalização é uma ocorrência rotineira em nossa população podendo contribuir para a polifarmácia, principalmente em idosos. Nesse sentido, a assistência farmacêutica tem um papel importante como estratégia de atenção à saúde para reduzir os impactos causados pela polimedicação (SILVA ACA, et al., 2019).

Embora a polifarmácia seja mais frequente em idosos, na amostra estudada observou-se prevalência de $8 \%$ na faixa etária de 18 a 59 anos. Um estudo holandês mostrou que, no período de 1999 a 2014, a maior ocorrência de polifarmácia foi em pacientes acima de 65 anos, porém, o aumento relativo foi maior em pessoas mais jovens (OKTORA MP, et al., 2019). Já há algum tempo pessoas com menos idade têm desenvolvido doenças crônicas em razão de hábitos de vida não saudáveis como sedentarismo, dieta não equilibrada, adesão ao tabagismo e consumo de bebida alcoólica. (MORAES ACF, et al., 2009).

Outras variáveis sociodemográficas como escolaridade, situação conjugal e renda familiar foram condições menos relevantes com relação à polifarmácia nos usuários do SUS entrevistados, diferentemente do observado em outros estudos (LOYOLA FILHO I, et al., 2006; RAMOS LR, et al., 2016; NASCIMENTO RCRM, et al., 2017; CAVALCANTI G, et al., 2017; ALMEIDA NA, et al., 2017). Embora 69,4\% dos entrevistados fossem mulheres, não houve associação positiva com o sexo feminino, diferentemente de outros estudos (GRIMMSMANN T, et al., 2009; COSTA GM, et al., 2017).

Houve maior prevalência de polifarmácia entre indivíduos que não estavam realizando atividade remunerada $(\mathrm{RP}=1,86)$. Uma das razões para não realizar atividade remunerada é a incapacidade laboral, principalmente na presença de multimorbidades, o que sugere maior utilização de serviços e medicamentos. Esta variável, presente em quase metade dos entrevistados, esteve fortemente associada na análise bivariada.

Várias doenças crônicas aumentam significativamente o risco de polifarmácia. Neste estudo, além da autopercepção negativa de saúde $(R P=2,0)$, em ordem crescente, houve associação significativa com depressão $(R P=1,47)$, artrite/artrose $(R P=1,50)$, doença cardíaca $(R P=1,60)$, diabetes mellitus $(R P=2,62)$ e hipertensão arterial sistêmica $(\mathrm{RP}=3,77)$. A maioria desses resultados era esperada e esses dados corroboram os da literatura (SILVEIRA EA, et al., 2014; RAMOS LR, et al., 2016; COSTA GM, et al., 2017; MARQUES PP, et al., 2019). São achados que conectam a ocorrência de doenças crônicas notadamente em idosos, em especial diabetes mellitus e hipertensão, e maior utilização de medicamentos que podem levar, inclusive, à interação medicamentosa (ROZENFELD S, et al., 2008; NASCIMENTO RCRM, et al., 2017).

Como também relatado em outros inquéritos, a polifarmácia não se associou a problemas crônicos como hipercolesterolemia e hipertrigliceridemia (RAMOS LR, et al., 2016; NASCIMENTO RCRM, et al., 2017). É possível que na amostra analisada muitas pessoas, embora com níveis sanguíneos elevados desses componentes, não utilizassem medicamentos para seu controle. Embora 65\% apresentassem excesso de peso, não foi significativa a relação com polifarmácia, como também ocorreu em outro estudo brasileiro, mas diferentemente da associação com obesidade encontrada no estudo FIBRA (SILVEIRA EA, et al., 2014; MARQUES PP, et al., 2019). Vale lembrar, no entanto, que essas condições são fatores de risco para a ocorrência de comorbidades como diabetesmellitus, hipertensão e surgimento de problemas cardiovasculares que, por sua vez, exigirão a utilização de medicamentos para o seu controle. 
Não houve associação com tabagismo, mas maior probabilidade do desfecho foi observada em participantes que não consumiam bebida alcoólica $(R P=1,41)$. Pacientes que utilizam muitos medicamentos podem apresentar maior chance de interação medicamentosa com bebidas alcoólicas (WANNMACHER L, 2007). Logo, seria recomendado que esses pacientes não bebessem.

Pelo que é mostrado neste estudo e na literatura disponível, há necessidade de medidas que visem promover o uso racional de medicamentos não somente em idosos, através de incentivo à melhoria dos hábitos de vida da população, como prática de atividades físicas, dieta equilibrada e ações educativas para a prevenção de doenças. Além disso, são necessárias medidas que disciplinem a prescrição, a dispensação e o consumo de medicamentos para obter a melhor resposta terapêutica possível. No contexto da APS, a assistência farmacêutica assume um papel importante como uma estratégia de atenção à saúde, desde a educação em saúde até o acompanhamento farmacoterapêutico, o que impactaria diretamente no uso racional dos medicamentos pela população (COSTA GM, et al., 2017; MARQUES PP, et al., 2019).

As informações em saúde são cada vez mais essenciais para o planejamento, a programação, 0 monitoramento e a gestão das intervenções em saúde coletiva e individual, principalmente quando se considera o contexto de mudanças do padrão epidemiológico, ampliação do conceito saúde-doença, bem como a incorporação das atividades de promoção da saúde (LOBO LB, 2015). As informações oriundas das fontes de dados secundários dos sistemas de informação em saúde são fundamentais, mas insuficientes para responder às necessidades da gestão. Daí a importância crescente de inquéritos populacionais, que além de avaliar as condições de saúde das populações, permitem ainda avaliar as políticas públicas implementadas, analisar temporalmente 0 acesso às políticas promotoras de saúde, comparar padrões de saúde entre áreas geográficas e calcular os custos das doenças para o sistema de saúde (WALDMAN EA, et al., 2008).

Como pontos fortes do estudo, destaca-se o poder estatístico adequado para as análises realizadas e a contribuição com o conhecimento sobre o tema ainda pouco explorado entre usuários da APS, sendo o primeiro realizado nessa região do estado. Além dos idosos, um grande número de usuários na faixa etária de 18 a 59 anos foram avaliados, sendo demonstrada uma associação importante com polifarmácia. Tal fato é preocupante e os dados do presente estudo podem contribuir para o aprimoramento de políticas públicas e a qualificação do atendimento na APS.

Como limitações, considera-se a natureza transversal, com possibilidade de viés de causalidade reversa entre algumas variáveis. Além disso, pode ter ocorrido viés de informação devido aos dados terem sido coletados por autorreferimento, a amostra não ter sido escolhida de maneira aleatória e não ter sido avaliado o perfil dos medicamentos utilizados pelos participantes. Estudos subsequentes devem avaliar, de forma mais abrangente, o consumo e o tipo de medicamentos utilizados pelos usuários, com abordagem na adesão, prescrição e forma de administração.

\section{CONCLUSÃO}

Pelos resultados deste estudo, a polifarmácia é uma realidade no contexto da APS, sobretudo em idosos. Este desfecho, que se mostrou associado a fatores comportamentais e de saúde, revela a necessidade de implementação de estratégias que alertem a população sobre os riscos e sensibilizem as equipes de saúde para melhorar a qualidade da assistência e reduzir essa situação preocupante em usuários da Atenção Primária à Saúde.

\section{REFERÊNCIAS}

1. ALMEIDA NA, et al. Prevalência e fatores associados à polifarmácia entre os idosos residentes na comunidade. Rev Bras Geriatr Gerontol, 2017; 20(1): 143-53.

2. ARRAIS PSD, et al. Prevalência da automedicação no Brasil e fatores associados. Rev Saúde Pública. 2016;50(supl 2):13s.

3. BRASIL. Ministério da Saúde (MS). Política Nacional de Atenção Básica. Disponível em: http://189.28.128.100/dab/docs/publicacoes/geral/pnab.pdf. Acessado em:13 de julho de 2020.

4. CAVALCANTI G, et al. Multimorbidade associado à polifarmácia e autopercepção negativa de saúde. Rev Bras Geriatr Gerontol, 2017; 20(5): 635-643. 
5. COSTA GM, et al. Fatores associados à polifarmacoterapia entre idosos assistidos pela estratégia saúde da família. Rev Bras Geriatr Gerontol,2017; 20(4): 528-537.

6. DOMINGUES PHF, et al.Prevalência da automedicação na população adulta do Brasil: revisão sistemática. Rev Saúde Pública, 2015;49:36.

7. FLORES LM, MENGUE SS. Uso de medicamentos por idosos em região do sul do Brasil. Rev Saúde Pública,2005; 39 (6): 924-29.

8. FLORES VB, BENVEGNú LA. Perfil de utilização de medicamentos em idosos da zona urbana de Santa Rosa, Rio Grande do Sul, Brasil. Cad Saúde Pública, 2008; 24(6): 1439-1446.

9. GRIMMSMANN T, HIMMEL W. Polypharmacy in primary care practices: an analysis using a large health insurance database. Pharmacoepidemiol Drug Saf, 2009; 18(12): 1206-13.

10. GUTHRIE B, et al. The rising tide of polypharmacy and drug-drug interactions: population database analysis 1995 2010. BMC Med, 2015; 13: 74.

11. INSTITUTO BRASILEIRO DE GEOGRAFIA E ESTATÍSTICA (IBGE). Censo demográfico. Disponível em: https://cidades.ibge.gov.br/brasil/rs/passo-fundo/panorama. Acessado em: 28 de abril de 2021.

12. LIPSCHITZ DA. Screening for nutritional status in the elderly. Prim Care, 1994; 21(1):55-67.

13. LOBO LB. Polifarmácia entre os idosos de Dourados, Mato Grosso do Sul: um estudo de base populacional. Dissertação (Mestrado em Ciências da Saúde). Faculdade de Ciências da Saúde. Universidade Federal da Grande Dourados, Dourados, 2015; 74 p.

14. LOYOLA FILHO Al, et al. Estudo epidemiológico de base populacional sobre uso de medicamentos entre idosos na Região Metropolitana de Belo Horizonte, Minas Gerais, Brasil. Cad Saude Publica, 2006; 22(12): 2657-67.

15. LUTZ BH, et al. Inadequação do uso de medicamentos entre idosos em Pelotas, RS. Rev Saúde Pública, 2017; 51: 52. 1-12.

16. MARIN MJS, et al. Caracterização do uso de medicamentos entre idosos de uma unidade do Programa Saúde da Família. Cad. Saúde Pública, 2008; 24(7): 1545-1555.

17. MARQUES PP, et al. Polifarmácia em idosos comunitários: resultados do estudo Fibra. Rev Bras Geriatr Gerontol, 2019; 22(5): e190118.

18. MASNOON N, et al. What is polypharmacy? A systematic review of definitions. BMC Geriatrics, 2017; 17: 230.

19. MORAES ACF, et al. Prevalência de síndrome metabólica em adolescentes: uma revisão sistemática. Cad. Saúde Pública, 2009; 25(6): 1195-1202.

20. NASCIMENTO RCRM, et al. Polifarmácia: uma realidade na atenção primária do Sistema Único de Saúde. Rev Saude Publica, 2017; 2(51): 1-12.

21. OKTORA MP, et al. Trends in polypharmacy and dispensed drugs among adults in the Netherlands as compared to the UnitedStates. PLoS One, 2019; 14(3): e0214240.

22. OLIVEIRA P.C, et al. Prevalência e Fatores Associados à Polifarmácia em Idosos Atendidos na Atenção Primária à Saúde em Belo Horizonte, MG. Cien Saude Colet [periódico na internet] (2019/Jul). Disponível em:http://www.cienciaesaudecoletiva.com.br/artigos/prevalencia-e-fatores-associados-a-polifarmacia-em-idososatendidos-na-atencao-primaria-a-saude-em-belo-horizonte-mg/17294?id=17294. Acessado em: 29 de abrilde 2021.

23. PASSO FUNDO. Prefeitura Municipal. Secretaria de Saúde. Plano Municipal de Saúde 2014-2017. Disponível em:http://www.pmpf.rs.gov.br/servicos/geral/files/portal/PMS_2014-2017.pdf. Acessado em: 28 de abril de 2021.

24. PEREIRA KG, et al. Polifarmácia em idosos: um estudo de base populacional. Rev Bras Epidemiol, 2017; 20(2): 335344.

25. RAMOS LR, et al. Polifarmácia e polimorbidade em idosos no Brasil: um desafio em saúde pública. Rev Saúde Pública, 2016; 50(supl 2): 9s.

26. ROZENFELD S, et al. Drug utilization and polypharmacy among the elderly: a survey in Rio de Janeiro City, Brazil. Rev Panam Salud Publica, 2008; 23(1): 34-43.

27. SECOLI SR, et al. Interações medicamentosas em pacientes coronariopatas. Rev Bras Cardiol, 2012; 25(1): 11-18.

28. SILVA ACA, et al. Assistência farmacêutica em casos de polifarmácia entre a população idosa. REAS/EJCH,, 2019; 28; e999.

29. SILVEIRA EA, et al. Polifarmácia, doenças crônicas e marcadores nutricionais em idosos. Rev Bras Epidemiol,2014; $17(4): 818-829$.

30. STEFANO ICA, et al. Uso de medicamentos por idosos: análise da prescrição, dispensação e utilização num município de porte médio do estado de São Paulo. Rev Bras Geriatr Gerontol,2017; 20(5): 681-692.

31. VICTORA CG, et al. The role of conceptual frameworks in epidemiological analysis: a hierarchical approach. International J Epidemiol, 1997; 26(1):224-227.

32. WALDMAN EA, et al. Inquéritos populacionais aspectos metodológicos, operacionais e éticos. Rev Bras Epidemiol, 2008; 11 (supl 1): 168-79.

33. WANNMACHER, L. Interações de medicamento com álcool: verdades e mitos. Ministério da Saúde.Brasília, v.4, n. 12, $2007 . \quad$ Disponível em: http://www.saudedireta.com.br/docsupload/1339892860v4n12_interacoes_medicamentosas.pdf.

34. WORLD HEALTH ORGANIZATION (WHO). AnthroPlus for personal computers Manual: Software for assessing growth of the world's children and adolescents. 2009. Disponível em: http://www.who.int/growthref/tools/. Acessado em: 14 de julho de 2020.

35. WORLD HEALTH ORGANIZATION (WHO). Medication Safety in Polypharmacy - Technical Report 2019. Disponível em: https://apps.who.int/iris/rest/bitstreams/1235792/retrieve. Acessado em: 24 de julho de 2020.

36. WORLD HEALTH ORGANIZATION (WHO). Physical status: the use and interpretation of anthropometry. Technical $\begin{array}{lllll}\text { Report } & \text { Series, } & \text { n. } & 854 . & \text { Disponível }\end{array}$ https://apps.who.int/iris/bitstream/handle/10665/37003/WHO_TRS_854.pdf?sequence=1\&isAllowed=y.Acessado em: 14 de julho de 2020 . 